\title{
Low paediatric tuberculosis case detection rate in Southern Mozambique
}

\author{
To the Editor:
}

Two core indicators adopted for evaluating tuberculosis (TB) control programmes are treatment outcome and case detection rate (CDR). While the former is easy to report, the CDR can only be estimated (calculated as notifications of new and relapse cases divided by estimated incidence). According to the World Health Organization (WHO), Mozambique has one of the lowest CDRs among the high TB burden countries (HBCs), with 37\% in 2013 [1]. In children, calculating CDR is even more challenging, given the difficulty in diagnosing TB and the lack of accurate estimates for paediatric incidence [2]. Several paediatric TB incidence estimates have been published recently [3-5], showing higher figures than those provided by WHO. The large variation in estimates and the lack of population-based data from HBCs (particularly from Sub-Saharan Africa) highlights the urgent need for new data to inform predictive models necessary to implement the "End TB Strategy" and achieve elimination [6]. The objective of this study was to calculate the paediatric CDR in Mozambique and to provide reference methodology and evidence for other countries.

The study was conducted in the Manhiça District, a high TB-HIV burden district in southern Mozambique [7], where the Manhiça Health Research Centre runs a health and demographic surveillance system (HDSS) [8]. It was a retrospective, population-based analysis, which estimated the TB CDR by comparing the routine TB incidence rate in children aged $<3$ years reported in the district between 2006 and 2010 with the incidence rate in the study area computed during a prospective study (the ITACA study: determination of the minimum incidence rate of tuberculosis in infants and children in the Manhiça District, Mozambique; October 2011-October 2012) [9]. The latter was considered as the "most accurate incidence rate estimate" available. Prior to the ITACA study, no routine active case finding was performed and bacteriological confirmation was mostly based on smear microscopy. During the ITACA period, all presumptive TB cases were identified through an active and passive case detection system. Children with TB-related symptoms and close contact with a sputum smear-positive TB patient were evaluated through physical and radiological examination, HIV and tuberculin skin testing, as well as smear microscopy and culture of both induced sputum and gastric aspirate samples $[9,10]$. For the purpose of this analysis, TB cases were defined as those who started anti-TB treatment. Relapse patients were included.

We calculated the TB incidence rate during the ITACA period as the number of cases in the HDSS area divided by the mid-year population at risk. Pre-ITACA incidence rates were calculated yearly using the number of TB cases in the whole district divided by the district mid-year population, using the Mozambican National Statistics Institute (INE) latest official census data (2007) and taking into account the estimated age-specific population growth for the whole period. Yearly confidence intervals were calculated assuming a Poisson distribution. A pooled incidence rate was calculated for the pre-ITACA period using a weighting scheme through a Poisson regression with random effects and jackknife 95\% CI. According to the 20002013 WHO data, there has been a slight increase in incidence during this time-frame in Mozambique (figure 1) [1]. Using a log-linear regression and WHO data, we estimated an overall $0.6 \%$ yearly increasing trend in TB incidence rate and applied this correction factor to the final CDR to mitigate overestimation.

A total of 217 TB cases aged $<3$ years were diagnosed in the pre-ITACA period (2006-2010), with a pooled incidence rate of 251 per 100000 people (95\% CI 227-276 per 100000). During ITACA, 57 TB cases aged $<3$ years initiated anti-TB treatment in the HDSS area, equivalent to an incidence rate of 615 per 100000 people (95\% CI 466-797 per 100000 people) (figure 1). The estimated CDR was 40.8\% (95\% CI 36.6-45.1\%), and $41.8 \%$ (95\% CI 37.2-46.4\%) after correction. In the hypothetical case of a $5 \%$ increase in the national incidence, sensitivity analysis showed that the CDR would increase to (49.1\%; 95\% CI 41.3-57.0\%).

The HIV prevalence among the study population was $47 \%$ and $46 \%$ during the pre-ITACA and ITACA periods, respectively. During the ITACA period, fewer patients aged $<1$ year initiated anti-TB treatment ( $8.8 \%$ versus $35.9 \%)$, more TB cases were extrapulmonary $(12.3 \%$ versus $7.4 \%)$ and the treatment success rate was significantly better $(82.5 \%$ versus $67.3 \%$; $\mathrm{p}=0.025)$.

To our knowledge, this is the first study providing estimates of TB under-detection using population-based data in the paediatric population in Africa and one of the few worldwide. We found a low CDR regardless of age and sex, which underscores the urgent need to close the gap in case detection and reporting, in order to better assess new control interventions $[6,11]$.

Our findings are in line with the low CDR for Mozambique reported by WHO [1]; however, the rate we report is probably a maximum, given that the ITACA incidence rate is a conservative estimate (single-day 


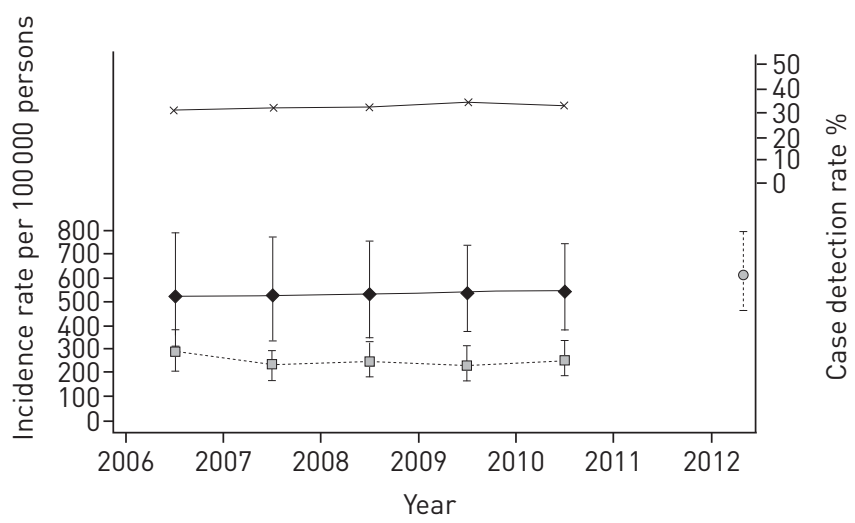

\footnotetext{
$\longrightarrow$ Case detection rate for all ages (WHO estimates)

...-.-..- Manhiça District incidence rate for age $<3$ years (pre-ITACA)

..........- Manhiça District incidence rate for age $<3$ years (ITACA)
}

FIGURE 1 Yearly tuberculosis incidence rate in the population aged <3 years in the Manhiça District. World Health Organization (WHO) estimates for all-age incidence rate and case detection rate for Mozambique are shown for the pre-ITACA period. ITACA: study for determination of the minimum incidence rate of tuberculosis in infants and children in the Manhiça District, Mozambique; October 2011-October 2012.

samples were obtained in contrast to the recommended 3-day consecutive sampling; contact tracing could not be fully implemented mainly due to difficulties in patient identification; and some TB cases could have been missed due to mortality prior to treatment initiation or transfers of severely ill patients to the tertiary reference hospital) [9].

The CDR higher than the 35\% estimated by DoDD et al. [5] for children aged $<15$ years in all HBCs (based on mathematical modelling of 2010 data) could be due to several reasons.

Given that Mozambique has one of the lowest CDRs among all HBCs and that under-estimation is more frequent in children aged $<5$ years, the difference observed between our CDR and the one reported by DoDD et al. [5] could support the hypothesis that the true CDR in Manhiça is probably lower than $40.8 \%$. Besides, TB incidence and CDR vary greatly across countries and regions (depending on the local epidemiology of TB/HIV and healthcare system characteristics, among others) and Manhiça could show an improved CDR compared with other settings.

Although under-ascertainment, under-reporting and under-diagnosis can all contribute to TB under-estimation [12], the latter is the most probable in Mozambique, where broad paediatric TB case definitions, lack of clear clinical algorithms, low referral rates and difficulties in obtaining samples all contribute to under-diagnosis. Under-reporting is not common, as the private sector has a small role in TB diagnosis and management. Under-ascertainment alone, although common in Manhiça, cannot explain the low CDR, given the small impact of the active case-finding component of this study.

This study has several limitations. First, the use of a historical control for the calculation of CDR may be imperfect as the incidence rate and CDR vary over time. Although we expected that the $0.6 \%$ yearly correction could compensate, we acknowledge that the extent of increase in incidence reported for adults may not be the same for children aged $<3$ years. Furthermore, we compared incidence rates calculated with different denominators (the intervention HDSS population, based on real annual census data for a smaller urban-shaped area versus the whole district population, based on projections from the INE 2007 census and considering age-specific annual population growth). We did, however, verify that the CDR did not vary substantially when we calculated the ITACA incidence based on INE district data (and using as denominator the percentage of the INE population belonging to HDSS).

This study provides a novel population-based CDR for paediatric TB in a HBC. Although this estimate is probably a maximum, differences in local detection rates can explain a higher CDR than those reported by others [1,5]. Given the multiple downstream ramifications of inaccurate estimates [13] and the high mortality of undiagnosed $\mathrm{TB}$ in younger children [14], this finding calls for urgent public health interventions to ensure that all TB cases are promptly identified and treated. 
Elisa López-Varela ${ }^{1,2}$, Orvalho Joaquim Augusto $^{1}$, Luis Guerra ${ }^{1}$, Durval Respeito ${ }^{1}$, Charfudin Sacoor ${ }^{1}$, Jahit Sacarlal $^{1}$, Giovanni Battista Migliori ${ }^{3}$, Giovanni Sotgiu ${ }^{4}$, Pedro L. Alonso ${ }^{1,2}$ and Alberto L. García-Basteiro ${ }^{1,2,5}$ ${ }^{1}$ Centro de Investigação em Saúde de Manhiça (CISM), Maputo, Mozambique. ${ }^{2}$ Instituto de Salud Global de Barcelona (ISGLOBAL), Barcelona, Spain. ${ }^{3}$ WHO Collaborating Centre for TB and Lung Diseases, Fondazione S. Maugeri, Care and Research Institute, Tradate, Italy. ${ }^{4}$ Clinical Epidemiology and Medical Statistics Unit, Dept of Biomedical Sciences, University of Sassari - Research, Medical Education and Professional Development Unit, AOU Sassari, Sassari, Italy.

${ }^{5}$ Amsterdam Institute for Global Health and Development, Academic Medical Centre, Amsterdam, The Netherlands.

Correspondence: Elisa López-Varela, Centro de Investigação em Saúde de Manhiça (CISM), Maputo, Mozambique.

E-mail: elisa.lopez@manhica.net

Received: Sept 012015 | Accepted after revision: Nov 092015 | First published online: Dec 232015

Conflict of interest: None declared.

Acknowledgements: The authors thank the participants and their families. The authors are grateful to the National TB Program (Maputo, Mozambique), Denise Naniche (ISGLOBAL, Barcelona, Spain), Alberto Bila Junior (CISM, Manhiça, Mozambique), health staff at the Manhiça District Hospital (Manhiça, Mozambique) and peripheral health centres for their contribution.

\section{References}

1 World Health Organization. Global Tuberculosis Report 2014. WHO/HTM/TB/2014.08. Geneva, World Health Organization, 2014. Available from: http://www.who.int/tb/publications/global_report/gtbr14_main_text.pdf

2 Marais BJ, Graham SM, Maeurer M, et al. Progress and challenges in childhood tuberculosis. Lancet Infect Dis 2013; 13: 287-289.

3 Murray CJ, Ortblad KF, Guinovart C, et al. Global, regional, and national incidence and mortality for HIV, tuberculosis, and malaria during 1990-2013: a systematic analysis for the Global Burden of Disease Study 2013. Lancet 2014; 384: 1005-1070.

4 Jenkins HE, Tolman AW, Yuen CM, et al. Incidence of multidrug-resistant tuberculosis disease in children: systematic review and global estimates. Lancet 2014; 383: 1572-1579.

5 Dodd PJ, Gardiner E, Coghlan R, et al. Burden of childhood tuberculosis in 22 high-burden countries: a mathematical modelling study. Lancet Glob Health 2014; 2: e453-e459.

6 Lönnroth K, Migliori GB, Abubakar I, et al. Towards tuberculosis elimination: an action framework for low-incidence countries. Eur Respir J 2015; 45: 928-952.

7 García-Basteiro AL, López-Varela E, Respeito D, et al. High tuberculosis burden among people living with HIV in southern Mozambique. Eur Respir J 2015; 45: 547-549.

8 Sacoor C, Nhacolo A, Nhalungo D, et al. Profile: Manhiça Health Research Centre (Manhiça HDSS). Int J Epidemiol 2013; 42: 1309-1318.

9 López-Varela E, Augusto OJ, Gondo $\mathrm{K}$, et al. Incidence of tuberculosis among young children in rural Mozambique. Pediatr Infect Dis J 2015; 34: 686-692.

10 García-Basteiro AL, López-Varela E, Augusto OJ, et al. Radiological findings in young children investigated for tuberculosis in Mozambique. PLoS One 2015; 10: e0127323.

11 Rudolf F, Wejse C. Tuberculosis case detection revisited: better testing might not improve outcomes. Lancet Glob Health 2015; 3: e424-e425.

12 Gibbons CL, Mangen MJ, Plass D, et al. Measuring underreporting and under-ascertainment in infectious disease datasets: a comparison of methods. BMC Public Health 2014; 14: 147.

13 Cruz AT, Starke JR. What's in a number? Accurate estimates of childhood tuberculosis. Lancet Glob Health 2014; 2: e432-e433.

14 Perez-Velez CM, Marais BJ. Tuberculosis in children. N Engl J Med 2012; 367: 348-361.

\section{Polymers of $Z \alpha_{1}$-antitrypsin are secreted in cell models of disease}

To the Editor:

The $\alpha_{1}$-antitrypsin $\left(\alpha_{1}-\mathrm{AT}\right)$ is a $52 \mathrm{kDa}$ glycoprotein that is predominantly synthesised in the liver and secreted into the circulation, where it protects the lungs from the enzyme neutrophil elastase. $\alpha_{1}$-AT deficiency $\left(\alpha_{1}\right.$-ATD) is caused by mutations in the $\alpha_{1}$-AT gene, with most cases resulting from homozygous inheritance of the Z allele (Glu342Lys). This leads to low levels of circulating $\alpha_{1}$-AT, uncontrolled elastase activity and emphysema [1]. The Z mutation destabilises the native $\alpha_{1}$-AT and causes the formation of aberrant polymers that accumulate within the endoplasmic reticulum (ER) of 